\title{
I. X-RAY MINERALOGY DATA FROM THE SOUTHEAST PACIFIC BASIN- LEG 35 DEEP SEA DRILLING PROJECT ${ }^{1}$
}

\author{
I. Zemmels and H.E. Cook, University of California, Riverside, California
}

\section{METHOD}

Semiquantitative determinations of the mineral composition of bulk samples, $2-20 \mu \mathrm{m}$, and $<2 \mu \mathrm{m}$ fractions were performed according to the methods described in the appendix of Volume 28.

The method of sample preparation, is as follows: Bulk samples are washed to remove seawater salts and are ground to less than $10 \mu \mathrm{m}$ under butanol. A portion of the sediment is decalcified in a sodium-acetate-buffered, acetic-acid solution ( $p \mathrm{H} \mathrm{4.5)}$. The residue is fractionated into $2-20 \mu \mathrm{m}$ and $<2 \mu \mathrm{m}$ samples by wet-sieving and centrifugation. The $2-20 \mu \mathrm{m}$ samples are ground to less than $10 \mu \mathrm{m}$. These three preparations are treated with trihexylamine acetate to expand the smectites. All samples are X-rayed as random powders.

The results of the $\mathrm{X}$-ray diffraction analysis are presented in Tables 1 to 4 . Table 5 contains the list of samples submitted for X-ray diffraction analysis, the subbottom depth of each sample which identifies the sample in Tables 1 to 4 , a color description, and a sediment description of each sample.

The sediment description is based on a classification devised in the DSDP X-ray Mineralogy Lab for rapid smear-slide analysis of deep-sea sediments. The classification comprises four major sediment types: detrital (d) consisting of fragmented silicates and clay minerals, biogenous (b) consisting of skeletal debris, authigenic (a) common examples of which are zeolitites and chert, and chemical (c) primarily the ironmanganese colloids. The sediment types are given equivalent rank. Operationally a sediment is detrital if volumetrically $d+b>a+c$ and $d>b$; biogenous if $d$ $+\mathrm{b}>\mathrm{a}+\mathrm{c}$ and $\mathrm{b}>\mathrm{d}$; authigenic if $\mathrm{a}+\mathrm{c}>\mathrm{d}+\mathrm{b}$ and $\mathrm{a}$ $>$ c; chemical if $a+c>d+b$ and $c>a$. Detrital sediments are further subdivided on the basis of texture into sand, silt, mud, and clay according to Folk's (1968) scheme.

Biogenous sediments are subdivided into siliceous ooze, calcareous ooze, and calcareous ooze in $25 \%$ increments of the components. The prefix bio is used when a biologic origin of the materials can be seen.

Authigenic and chemical sediments are given only gross descriptive terms such as chert or iron manganese colloid.

Components of other groups which appear in the major sediment type are acknowledged by modifiers to the sediment name according to the following scheme: components in concentrations of $2 \%-10 \%$ are used as adjectives or in conjunction with "bearing" (i.e., clayey and

\footnotetext{
IInstitute of Geophysics and Planetary Physics, University of California, Riverside, California, Contribution No. 75-5.
}

clay-bearing are synonymous), 10\%-25\% concentrations are termed "rich," $25 \%-50 \%$ concentrations are termed "abundant."

Mudrocks were named according to the classification of Blatt et al. (1972) which uses textural criteria for silt, mud, and clay and differentiates between fissile and nonfissile rocks. Thus, if abundant silt is visible, we have silt-shale or siltstone; if a grittiness is felt when chewed or scraped, we have mud-shale or mudstone; if no grittiness is felt, we have clay-shale or claystone. The term "argillite" is reserved for nonfissile mudrocks which show signs of incipient metamorphism.

A portion of the samples were run on Norelco diffraction equipment belonging to the Earth Sciences Department at the University of California, Riverside, because the DSDP equipment was inoperable part of the time. The two sets of data are compatible but the analytical precision of the Norelco-run data is somewhat lower. The Norelco-run data can be recognized in Tables 1 to 4 by the fact that no amorphous content is reported.

The percent amorphous is a measure of the weight fraction of amorphous material in each sample which commonly consists of biogenic silica, volcanic glass, palagonite, allophane, and organic material. The amorphous content is calculated from the total diffuse scatter of the sample. The method of calculation assumes that the diffuse scatter in excess of the diffuse scatter from the crystalline materials is proportioned to the amorphous content. The diffuse scatter of the crystalline minerals is determined from the mineral calibration standards (Cook et al., 1975). Ideally, the amorphous content varies between zero and $100 \%$, but, in cases where the minerals in the sample have a higher degree of crystallinity than the calibration standards, negative values can result. The negative values are reported as blanks; these samples can be assumed to contain little or no amorphous material.

The crystalline minerals are quantified by the method of mutual ratios using peak heights and concentration factors derived from ratioing the diagnostic peaks of minerals with the major peak of quartz. Unquantifiable minerals, i.e., unidentified minerals and minerals for which standards are not available, are tentatively quantified using a hypothetical concentration factor of 3.0 which is applied to the major peak of the mineral. The concentrations of the quantifiable minerals are summed to $100 \%$. The amorphous content and the unquantifiable minerals are not included in the total. The unquantifiable minerals are reported on a qualitative scale as trace (less than $5 \%$ ), present (5\%-25\%), abundant $25 \%$ $65 \%$ ), and major (greater than $65 \%$ ).

The precision of the mineral determination is approximately \pm 1 weight percent of the amount present. Because of crystallinity differences between the mineral 
TABLE 1

Results of X-Ray Diffraction Analysis From Site 322

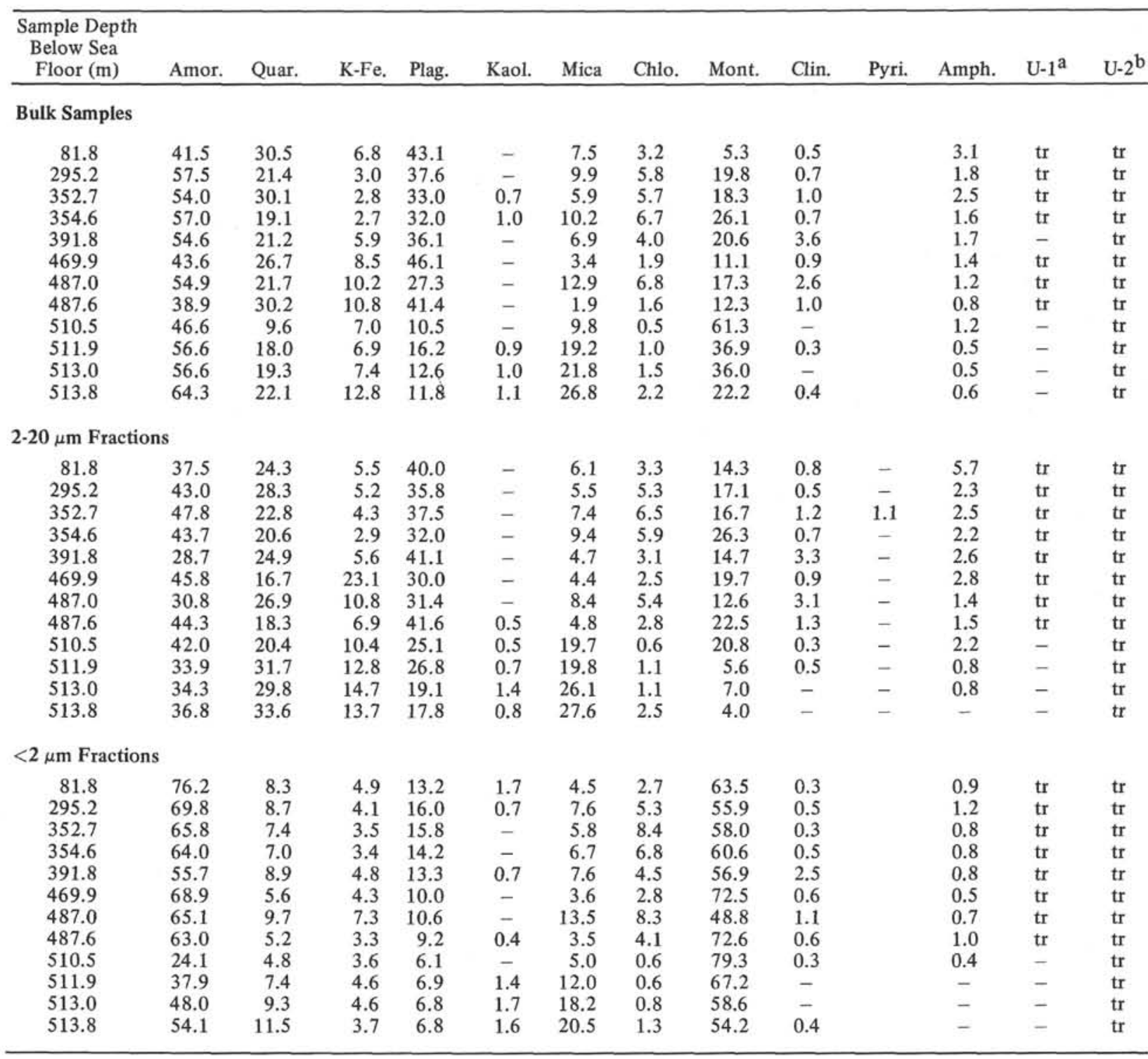

${ }^{a} U-1$ persistent peak at $9.5 \AA$. Intensity of peak was not observed to be dependent on any other minerals identified.

${ }^{b_{U}}$-2 peak at $2.53 \AA$, possibly magnetite. Optical examination indicates its presence, but secondary peaks were not detected.

calibration standards and the minerals in the samples and also diffraction peak interferences, the accuracy of the reported concentrations is often less than the precision of the method allows. In terms of the reported concentration, smectites may vary $\pm 50 \%$; micas, chlorites, cristobalite, tridymite. geothite may vary $\pm 20 \%$; kaolinite, amphibole, augite, the feldspars the zeolites, palygorskite, sepiolite, apatite may vary $\pm 10 \%$; the minerals which have stable crystal lattices and are not members of solid-solution series (or typically have limited crystal-lattice substitution in the sedimentary environment) such as quartz, low-magnesium calcite, aragonite, dolomite, rhodochrosite, siderite, gibbsite, talc, barite, anatase, gypsum, anhydrite, halite, pyrite, hematite, magnetite, will vary less than $\pm 5 \%$.

The user of the X-ray mineralogy data should bear in mind that (1) the reported values are relative concen- trations and that some adjustment has to be made for the amorphous content and the unquantifiable minerals to obtain the absolute concentrations, (2) in a homogeneous system of minerals, the mineral concentration trends are reliable because of the precision, but when comparing mineral concentrations between different geographic regions or lithologic units additional information regarding the crystallinity of the minerals is required, (3) the representativeness of the samples selected for X-ray diffraction analysis is the responsibility of the shipboard scientists and any questions pertaining to this aspect should be directed to them.

DRILLING AND MUD USAGE

Drilling mud, containing montmorillonite and barite, was used in Hole 325 between Cores 8 and 9 . The sample 
TABLE 2

Results of X-Ray Diffraction Analysis From Site 323

\begin{tabular}{|c|c|c|c|c|c|c|c|c|c|c|c|c|c|c|}
\hline $\begin{array}{l}\text { Sample Depth } \\
\text { Below Sea } \\
\text { Floor (m) }\end{array}$ & Amor. & Calc. & Dolo. & Quar. & Cris. & $\mathrm{K}-\mathrm{Fe}$. & Plag. & Kaol. & Mica & Chlo. & Goet. & Mont. & Trid. & Clin. \\
\hline Bulk Samples & & & & & & & & & & & & & & \\
\hline 76.6 & 33.8 & - & - & 45.3 & - & 16.1 & 28.8 & - & 5.6 & 1.7 & - & 2.6 & - & - \\
\hline 79.3 & 62.0 & - & - & 19.5 & - & 3.7 & 20.0 & - & 22.1 & 6.2 & - & 26.7 & - & 0.7 \\
\hline 161.8 & 66.0 & - & - & 16.0 & - & 9.7 & 14.3 & 0.8 & 24.9 & 1.3 & - & 31.7 & - & 0.3 \\
\hline 162.2 & 54.2 & - & - & 16.9 & - & 5.7 & 15.9 & 0.9 & 15.9 & 0.6 & - & 42.5 & - & 0.6 \\
\hline 258.7 & 32.2 & - & - & 42.7 & - & 13.2 & 35.7 & - & 2.6 & 1.5 & - & 2.5 & - & 0.7 \\
\hline 258.9 & 46.9 & - & - & 13.2 & - & 7.7 & 15.1 & - & 11.7 & - & - & 51.1 & - & 0.5 \\
\hline 333.4 & 57.8 & - & - & 15.6 & - & 2.3 & 19.0 & 0.4 & 20.4 & 0.6 & - & 40.0 & - & 0.8 \\
\hline 341.5 & 59.9 & - & - & 15.5 & - & 3.9 & 18.7 & 0.4 & 17.3 & 0.3 & - & 42.5 & - & 0.3 \\
\hline 341.5 & 48.5 & - & - & 13.5 & - & 7.4 & 17.0 & 0.7 & 14.9 & - & - & 45.3 & - & 0.5 \\
\hline 341.5 & 62.8 & - & - & 23.9 & - & 9.1 & 27.3 & - & 18.9 & 9.4 & - & 10.3 & - & 0.4 \\
\hline 342.9 & 60.5 & - & - & 13.0 & - & 8.1 & 13.6 & 0.7 & 19.7 & 0.2 & - & 43.8 & - & 0.2 \\
\hline 343.0 & 62.6 & - & - & 14.6 & - & 4.5 & 15.1 & 0.8 & 19.9 & - & - & 43.9 & - & 0.3 \\
\hline 363.6 & 48.6 & - & - & 16.2 & - & 8.5 & 17.1 & 0.4 & 16.2 & 2.2 & - & 38.2 & - & 0.3 \\
\hline 409.1 & 38.0 & - & - & 12.9 & - & 5.6 & 15.8 & 0.3 & 20.8 & 1.5 & - & 42.1 & - & 0.2 \\
\hline 409.2 & 67.8 & - & - & 8.5 & 57.5 & 2.6 & 6.2 & 0.2 & 7.8 & 1.4 & - & 2.3 & 13.4 & - \\
\hline 409.4 & 70.2 & - & - & 22.1 & 15.8 & 6.3 & 17.0 & - & 21.9 & 6.6 & - & 8.2 & 1.7 & 0.4 \\
\hline 458.0 & 60.5 & - & - & 24.1 & - & 17.4 & 21.3 & - & 15.9 & 2.4 & - & 17.4 & - & - \\
\hline 458.3 & 49.6 & - & - & 6.2 & 70.9 & 2.8 & 4.7 & - & 4.7 & 0.7 & - & - & 10.0 & - \\
\hline 504.2 & 46.2 & - & - & 22.3 & - & 9.4 & 22.4 & - & 11.6 & 3.4 & - & 29.9 & - & 0.4 \\
\hline 505.7 & 51.6 & - & - & 21.8 & - & 7.2 & 20.8 & 0.5 & 18.7 & 2.1 & - & 26.9 & - & 0.7 \\
\hline 507.3 & 52.3 & - & - & 20.1 & - & 11.4 & 18.6 & 0.5 & 19.9 & 3.5 & - & 24.8 & - & 0.7 \\
\hline 551.2 & 57.2 & - & - & 24.9 & - & 11.4 & 16.3 & 0.6 & 27.0 & 7.6 & - & 11.9 & - & 0.4 \\
\hline 600.2 & 39.6 & - & - & 32.1 & - & 10.0 & 18.5 & - & 9.9 & 1.2 & - & 27.8 & - & 0.5 \\
\hline 600.3 & 39.8 & - & - & 28.4 & - & 9.1 & 17.8 & - & 19.9 & 1.1 & - & 23.7 & - & - \\
\hline 600.6 & 41.8 & - & - & 19.2 & - & 8.7 & 9.6 & 1.4 & 29.1 & 7.3 & - & 24.9 & - & - \\
\hline 625.4 & 46.3 & - & - & 26.4 & - & 12.0 & 11.7 & 1.3 & 15.0 & 4.0 & - & 29.6 & - & - \\
\hline 638.4 & 47.1 & - & - & 11.3 & - & 7.7 & 7.3 & 0.9 & 9.9 & 2.5 & 5.8 & 54.6 & - & - \\
\hline 656.6 & 41.7 & - & - & 11.3 & - & 13.7 & 8.9 & 1.2 & 8.0 & 1.9 & 6.5 & 48.4 & - & - \\
\hline 657.9 & 53.3 & - & - & 12.6 & - & 14.5 & 8.6 & 2.0 & 7.9 & 1.6 & 8.2 & 44.4 & - & - \\
\hline 659.5 & 43.1 & - & - & 11.9 & - & 13.5 & 6.9 & 1.8 & 7.4 & 2.0 & 6.7 & 49.7 & - & - \\
\hline 660.7 & 45.3 & - & - & 10.2 & - & 12.6 & 3.7 & 2.3 & 5.3 & 1.6 & 7.2 & 57.2 & - & - \\
\hline 662.4 & 59.8 & - & - & 12.3 & - & 18.3 & 3.7 & 2.3 & 7.9 & 2.6 & 10.3 & 42.6 & - & - \\
\hline 663.5 & 64.8 & 73.1 & - & 3.2 & - & 3.9 & 1.3 & - & 2.7 & - & 3.2 & 12.7 & - & - \\
\hline 665.4 & 35.5 & 55.3 & 0.2 & 3.8 & - & 4.5 & 1.0 & - & - & - & - & 35.2 & - & - \\
\hline 667.8 & 42.9 & - & - & 8.1 & - & 11.8 & 3.8 & 0.8 & 4.1 & 0.3 & - & 71.1 & - & - \\
\hline 669.7 & 16.3 & - & - & 5.8 & - & 18.4 & 2.1 & - & 6.7 & - & - & 63.6 & - & 3.5 \\
\hline 695.7 & 28.6 & - & - & 10.7 & - & 15.8 & 3.1 & - & 8.6 & 0.3 & - & 58.0 & - & 3.5 \\
\hline 697.0 & 54.7 & - & - & 12.8 & - & 14.5 & 6.4 & - & 11.0 & 0.3 & - & 44.5 & - & 10.5 \\
\hline 698.0 & 30.1 & - & - & 13.7 & - & 5.7 & 9.2 & - & 5.9 & - & - & 46.2 & - & 18.8 \\
\hline 699.0 & 36.1 & - & - & 14.7 & - & 9.3 & 9.6 & - & 7.6 & - & - & 45.6 & - & 12.7 \\
\hline $2-20 \mu \mathrm{m}$ Frac & & & & & & & & & & & & & & \\
\hline 76.6 & 31.7 & & & 39.3 & - & 15.8 & 32.1 & 0.9 & 4.9 & 2.1 & - & 2.3 & - & 0.6 \\
\hline 79.3 & 39.3 & & & 25.6 & - & 7.0 & 25.9 & 0.6 & 19.6 & 7.3 & - & 12.2 & - & 0.4 \\
\hline 161.8 & 49.7 & & & 27.3 & - & 11.4 & 22.2 & 1.3 & 22.9 & 1.5 & - & 11.5 & - & 0.5 \\
\hline 162.2 & 42.9 & & & 30.0 & - & 13.6 & 26.5 & - & 17.0 & 1.1 & - & 10.6 & - & 0.5 \\
\hline 258.7 & 43.0 & & & 38.8 & - & 10.5 & 36.6 & - & 4.9 & 2.9 & - & 4.6 & - & 0.6 \\
\hline 258.9 & 35.3 & & & 25.0 & - & 10.2 & 25.1 & - & 15.8 & - & - & 22.1 & - & 0.4 \\
\hline 333.4 & 38.7 & & & 26.7 & - & 7.2 & 32.7 & - & 18.3 & - & - & 12.4 & - & 1.3 \\
\hline 341.5 & 46.6 & & & 21.5 & - & 7.0 & 24.6 & - & 18.0 & 0.8 & - & 27.2 & - & 0.4 \\
\hline 341.5 & 43.5 & & & 24.1 & - & 10.0 & 26.9 & - & 14.0 & - & - & 23.2 & - & 0.4 \\
\hline 341.5 & 41.3 & & & 24.2 & - & 9.8 & 24.9 & 0.6 & 13.7 & 11.8 & - & 12.8 & - & 0.8 \\
\hline 342.9 & 59.1 . & & & 22.9 & - & 6.6 & 20.6 & - & 19.8 & - & - & 28.5 & - & 0.4 \\
\hline 343.0 & 65.1 & & & 24.7 & - & 8.3 & 20.9 & - & 21.6 & 0.9 & - & 21.7 & - & 0.4 \\
\hline 363.6 & 34.4 & & & 24.3 & - & 12.1 & 22.9 & - & 18.4 & 3.6 & - & 17.2 & - & 0.4 \\
\hline 409.1 & 36.4 & & & 23.5 & - & 9.6 & 22.9 & - & 22.3 & 3.1 & - & 18.0 & - & - \\
\hline 409.2 & 32.8 & & & 24.9 & 41.3 & 6.8 & 11.9 & - & 6.3 & 1.4 & - & - & 7.4 & - \\
\hline 409.4 & N.D. C & & & 34.9 & - & 9.5 & 21.6 & 0.8 & 17.6 & 7.3 & - & 8.2 & - & - \\
\hline 458.0 & 31.5 & & & 30.9 & - & 6.9 & 28.6 & - & 13.4 & 3.9 & - & 14.3 & - & 0.5 \\
\hline 458.3 & N.D. & & & 18.1 & 54.8 & 4.3 & -9.6 & - & 4.8 & - & - & - & 8.4 & - \\
\hline 504.2 & 24.1 & & & 28.5 & - & 8.8 & 27.9 & - & 14.1 & 4.7 & - & 14.8 & - & 0.5 \\
\hline 505.7 & 26.2 & & & 32.4 & - & 10.2 & 23.6 & - & 16.2 & 3.0 & - & 13.2 & - & 0.5 \\
\hline 507.3 & N.D. & & & 32.0 & - & 12.8 & 24.9 & - & 13.8 & 4.1 & - & 11.1 & - & 0.5 \\
\hline 551.2 & N.D. & & & 37.5 & - & 12.1 & 19.9 & - & 20.4 & 7.9 & - & 2.2 & - & - \\
\hline 600.2 & N.D. & & & 40.7 & - & 14.8 & 24.5 & - & 11.3 & 2.3 & - & 4.8 & - & 0.7 \\
\hline 600.3 & N.D. & & & 39.4 & - & 15.8 & 26.0 & - & 14.9 & 0.8 & - & 2.4 & - & 0.7 \\
\hline 600.6 & N.D. & & & 47.9 & - & 13.6 & 17.7 & - & 15.2 & 5.7 & - & - & - & - \\
\hline 624.2 & N.D. & & & 39.3 & - & 13.1 & 18.3 & - & 19.7 & 5.9 & - & 3.7 & - & - \\
\hline 625.4 & N.D. & & & 47.0 & - & 13.2 & 16.8 & 0.7 & 15.2 & 4.7 & - & 2.4 & - & - \\
\hline 638.4 & N.D. & & & 31.1 & - & 16.9 & 18.2 & 0.7 & 14.5 & 4.1 & 4.2 & 10.2 & - & - \\
\hline 656.6 & N.D. & & & 32.3 & - & 23.2 & 17.7 & 1.0 & 10.2 & 2.6 & 5.4 & 7.8 & - & - \\
\hline 657.9 & N.D. & & & 28.2 & - & 24.9 & 15.3 & 1.8 & 11.6 & 1.7 & 5.6 & 10.8 & - & - \\
\hline 659.6 & N.D. & & & 31.2 & - & 24.1 & 13.1 & 1.4 & 10.3 & 3.1 & 5.5 & 11.4 & - & - \\
\hline 660.7 & N.D. & & & 28.9 & - & 28.6 & 10.3 & 2.4 & 10.5 & 2.5 & 6.1 & 10.8 & - & - \\
\hline 662.4 & N.D. & & & 31.0 & - & 30.5 & 7.9 & 0.4 & 9.6 & 4.5 & 6.2 & 9.9 & - & - \\
\hline 663.5 & N.D. & & & 31.0 & - & 33.0 & 5.9 & 0.4 & 9.8 & 3.8 & 6.9 & 9.1 & - & - \\
\hline 665.4 & N.D. & & & 30.8 & - & 33.6 & 8.6 & 0.4 & 6.5 & 2.0 & - & 18.2 & - & - \\
\hline 667.8 & N.D. & & & 32.3 & - & 37.6 & 5.6 & 0.7 & 9.2 & 0.8 & - & 13.8 & - & - \\
\hline 669.7 & N.D. & & & 27.8 & - & 40.8 & 10.2 & - & 7.9 & - & - & 7.4 & - & 5.9 \\
\hline 695.7 & N.D. & & & 30.8 & - & 29.9 & 15.7 & - & 12.0 & - & - & 6.7 & - & 4.8 \\
\hline 697.0 & N.D. & & & 31.3 & - & 25.0 & 11.8 & - & 11.4 & - & - & 7.3 & - & 13.2 \\
\hline 698.0 & N.D. & & & 29.9 & - & 12.1 & 22.0 & - & 7.9 & - & - & 4.2 & - & 23.9 \\
\hline 699.0 & N.D. & & & 32.5 & - & 16.3 & 16.2 & - & 9.7 & - & - & 5.1 & - & 20.3 \\
\hline
\end{tabular}


TABLE 2 - Continued

\begin{tabular}{|c|c|c|c|c|c|c|c|c|c|c|c|c|c|c|c|c|c|c|}
\hline $\begin{array}{l}\text { Sample Depth } \\
\text { Below Sea } \\
\text { Floor }(\mathrm{m})\end{array}$ & Amor. & Calc. & Dolo. & Quar. & Cris. & $\mathrm{K}-\mathrm{Fe}$. & Plag. & Kaol. & Mica & Chlo. & Goet. & Mont. & Trid. & Clin. & Gibb. & Amph. & $\mathrm{U}-1^{\mathrm{a}}$ & $\mathrm{U}-2^{\mathrm{b}}$ \\
\hline \multicolumn{19}{|c|}{$<2 \mu \mathrm{m}$ Fractions } \\
\hline 76.6 & 58.2 & & & 9.1 & - & 8.1 & 11.9 & 1.4 & 12.8 & 7.8 & - & 47.7 & - & 0.6 & & 0.6 & $\mathrm{tr}$ & - \\
\hline 79.3 & 64.0 & & & 8.8 & - & 3.8 & 10.5 & 2.0 & 15.8 & 7.6 & - & 51.5 & - & - & & - & - & - \\
\hline 161.8 & 63.0 & & & 7.9 & - & 2.8 & 8.7 & 2.6 & 13.8 & 0.9 & - & 62.6 & - & 0.3 & & 0.4 & $\operatorname{tr}$ & - \\
\hline 162.2 & 54.7 & & & 7.1 & - & 3.6 & 8.8 & 1.1 & 13.0 & 0.3 & - & 65.1 & - & 0.5 & & 0.4 & $\operatorname{tr}$ & tr \\
\hline 258.7 & N.D. & & & 8.4 & - & 7.3 & 11.2 & 2.1 & 19.0 & 11.0 & - & 35.5 & - & 4.9 & & 0.5 & tr & - \\
\hline 258.9 & 34.9 & & & 4.5 & - & 3.9 & 5.0 & 0.4 & 6.5 & - & - & 78.5 & - & 0.3 & & - & $\mathrm{tr}$ & - \\
\hline 333.4 & N.D. & & & 9.0 & - & 9.0 & 13.0 & - & 12.6 & - & - & 55.4 & - & 1.0 & & - & - & - \\
\hline 341.5 & N.D. & & & 7.0 & - & 6.2 & 10.0 & - & 9.7 & - & - & 67.2 & - & - & & - & - & - \\
\hline 341.5 & 36.6 & & & 5.0 & - & 5.0 & 7.5 & - & 7.0 & 0.3 & - & 74.6 & - & 0.3 & & 0.4 & tr & - \\
\hline 341.5 & 59.8 & & & 10.3 & - & 4.5 & 13.5 & - & 14.1 & 8.2 & - & 48.8 & - & 0.4 & & 0.3 & $\mathrm{tr}$ & - \\
\hline 342.9 & 51.9 & & & 6.6 & - & 5.5 & 9.0 & - & 11.5 & 0.5 & - & 66.3 & - & 0.2 & & 0.4 & tr & - \\
\hline 343.0 & 58.8 & & & 7.8 & - & 7.3 & 10.3 & 0.3 & 14.8 & 0.2 & - & 58.6 & - & 0.2 & & 0.4 & tr & tr \\
\hline 363.6 & 39.2 & & & 5.1 & - & 4.1 & 7.1 & 0.4 & 11.5 & 0.5 & - & 70.6 & - & 0.2 & & 0.4 & $\mathrm{tr}$ & - \\
\hline 409.1 & 29.5 & & & 4.7 & - & 4.7 & 7.0 & 0.3 & 10.3 & 0.3 & - & 72.2 & - & 0.2 & & 0.4 & - & tr \\
\hline 409.2 & 63.2 & & & 5.7 & 65.1 & 3.2 & 5.7 & 0.4 & 5.0 & 1.2 & - & 2.1 & 11.7 & - & & - & - & - \\
\hline 409.4 & 55.5 & & & 6.7 & 36.9 & 4.2 & 8.5 & 0.4 & 13.8 & 3.6 & - & 21.4 & 4.3 & 0.3 & & - & - & - \\
\hline 458.0 & 50.8 & & & 6.7 & - & 6.1 & 10.1 & 0.4 & 10.3 & 1.4 & - & 64.8 & - & 0.2 & & - & tr & - \\
\hline 458.3 & 56.3 & & & 3.2 & 66.7 & 2.1 & 2.8 & - & 5.5 & - & - & 7.0 & 12.7 & - & & - & - & - \\
\hline 504.2 & 38.4 & & & 6.1 & - & 6.7 & 8.7 & 0.3 & 9.3 & 2.2 & - & 66.2 & - & 0.5 & & - & - & - \\
\hline 505.7 & 40.3 & & & 6.3 & - & 6.1 & 6.6 & - & 9.4 & 0.8 & - & 70.3 & - & 0.5 & & - & - & - \\
\hline 507.3 & 46.9 & & & 12.9 & - & 4.6 & 13.6 & 0.3 & 12.7 & 2.2 & - & 53.5 & - & 0.2 & & - & - & - \\
\hline 551.2 & 49.5 & & & 11.2 & - & 8.1 & 9.4 & 0.3 & 19.3 & 4.8 & - & 46.5 & - & 0.4 & & - & - & - \\
\hline 600.2 & 18.1 & & & 4.5 & - & 2.9 & 4.2 & - & 8.0 & 0.6 & - & 79.7 & - & - & & - & - & - \\
\hline 600.3 & 21.6 & & & 6.3 & - & 2.8 & 3.7 & - & 11.8 & 0.9 & - & 75.5 & - & - & & - & - & - \\
\hline 600.6 & 36.5 & & & 9.8 & - & 6.8 & 6.0 & - & 23.1 & 6.1 & - & 47.7 & - & 0.2 & & 0.3 & - & - \\
\hline 624.2 & 44.1 & & & 7.7 & - & 8.0 & 5.2 & 1.0 & 12.0 & 2.2 & - & 63.9 & - & - & & - & - & - \\
\hline 625.4 & 34.1 & & & 7.0 & - & 6.5 & 5.0 & 0.7 & 10.8 & 3.0 & - & 57.1 & - & - & & - & - & - \\
\hline 638.4 & 31.1 & & & 3.2 & - & 2.3 & 1.5 & 0.8 & 2.1 & 1.0 & 5.0 & 84.1 & - & - & & - & - & - \\
\hline 656.6 & 34.5 & & & 5.3 & - & 5.7 & 2.8 & 1.1 & 3.0 & 0.5 & 6.9 & 74.7 & - & - & & - & - & - \\
\hline 657.9 & 44.4 & & & 4.4 & - & 6.6 & 1.9 & 0.8 & 2.0 & 0.5 & 7.2 & 76.5 & - & - & & - & - & - \\
\hline 659.5 & 37.9 & & & 6.7 & - & 7.5 & 4.0 & 0.7 & 4.8 & 0.9 & 4.4 & 71.1 & - & - & & - & - & - \\
\hline 660.7 & 40.7 & & & 5.1 & - & 6.4 & 1.8 & 1.5 & 2.0 & 0.9 & 6.9 & 75.5 & - & - & & - & - & - \\
\hline 662.4 & 46.0 & & & 8.0 & - & 10.8 & 2.9 & 1.7 & 5.3 & & 6.2 & 63.8 & - & - & & - & - & - \\
\hline 663.5 & 47.2 & & & 3.9 & - & 5.4 & 1.0 & 1.2 & 2.1 & 1.0 & 6.3 & 79.1 & - & - & & - & - & - \\
\hline 665.4 & 12.9 & & & 1.5 & - & 2.4 & 1.0 & 0.4 & 1.1 & 0.3 & - & 93.2 & - & - & & - & - & - \\
\hline 667.8 & 2.8 & & & 3.0 & - & 3.8 & 2.0 & - & 1.1 & - & - & 90.1 & - & - & & - & - & - \\
\hline 669.7 & 39.5 & & & 1.5 & - & 2.4 & 0.5 & - & 1.1 & - & - & 93.9 & - & 0.6 & & - & - & - \\
\hline 695.7 & 42.8 & & & 4.3 & - & 5.9 & 2.4 & - & 3.1 & - & - & 83.1 & - & 1.3 & & - & - & - \\
\hline 697.0 & 46.3 & & & 3.0 & - & 3.1 & 2.5 & - & 3.2 & - & - & 86.8 & - & 1.4 & & - & - & - \\
\hline 698.0 & 37.7 & & & 2.5 & - & 1.6 & 1.0 & - & 2.2 & - & - & 91.3 & - & 1.4 & & - & - & - \\
\hline 699.0 & 44.2 & & & 3.7 & - & 3.0 & 1.5 & - & 2.1 & - & - & 86.9 & - & 2.8 & & - & - & - \\
\hline
\end{tabular}

${ }^{a} U-1$ Persistent peak at 9.5A. Intensity of peak was not observed to be dependent on any other minerals identified.

${ }^{b}$ U-2 Peak at 2.53 A, possibly magnetite. Optical examination indicates its presence, but secondary peaks were not detected.

$\mathrm{C}_{\mathrm{N} . \mathrm{D} .}=$ no data.

submitted for X-ray diffraction analysis from Core 9 (depth $643.6 \mathrm{~m}$ ) which may have been directly exposed to the drilling mud does not contain an inordinate amount of montmorillonite and contains no barite.

\section{ACKNOWLEDGMENTS}

The writers wish to acknowledge the excellent work of David Berry and Pat Harrold in the interpretation of X-ray diffraction data, of Paul D. Johnson in X-ray data acquisition and data processing, and of Tom W. Halverson, Jr., in sample preparation.

\section{REFERENCES}

Blatt, H., Middleton, G.V., and Murray, R.C., 1972. The origin of sedimentary rocks: New York (Prentice Hall).

Cook, H.E., Johnson, P.D., Matti, J.C., and Zemmels, I., 1975. Methods of sample preparation and $\mathrm{x}$-ray diffraction data analysis, $x$-ray mineralogy laboratory, Deep Sea Drilling Project, University of California, Riverside. In Hayes, D.E., Frakes, L.A., et al., 1975. Initial Reports of the Deep Sea Drilling Project, Volume 28: Washington (U.S. Government Printing Office), p. 999-1008.

Folk, R.L., 1968. Petrology of sedimentary rocks (syllabus): Austin (Hemphill's). 
TABLE 3

Results of X-Ray Diffraction Analysis From Site 324

\begin{tabular}{|c|c|c|c|c|c|c|c|c|c|c|c|}
\hline $\begin{array}{l}\text { Sample Depth } \\
\text { Below Sea } \\
\text { Floor }(\mathrm{m})\end{array}$ & Amor. & Quar. & $\mathrm{K}-\mathrm{Fe}$. & Plag. & Kaol. & Mica & Chlo. & Mont. & Clin. & Amph. & $\mathrm{U}-1^{\mathrm{a}}$ \\
\hline \multicolumn{12}{|l|}{ Bulk Samples } \\
\hline 12.3 & 64.2 & 15.7 & 9.3 & 19.2 & - & 18.4 & 5.1 & 30.3 & 0.3 & 1.8 & $\operatorname{tr}$ \\
\hline 15.4 & 60.5 & 24.6 & 13.9 & 23.5 & 1.2 & 14.6 & 5.3 & 15.1 & 0.4 & 1.4 & $\operatorname{tr}$ \\
\hline 49.2 & 58.4 & 36.8 & 25.8 & 24.2 & 0.8 & 6.8 & 1.4 & 4.2 & - & - & - \\
\hline 50.2 & 54.4 & 22.2 & 15.4 & 15.3 & 4.3 & 24.3 & 4.2 & 13.3 & 0.4 & 0.6 & tr \\
\hline 50.6 & 48.9 & 27.5 & 16.2 & 21.9 & 0.7 & 12.2 & 3.5 & 16.2 & 0.5 & 1.4 & tr \\
\hline 79.3 & 49.2 & 21.8 & 15.0 & 16.9 & 2.6 & 19.5 & 5.4 & 18.2 & - & 0.6 & - \\
\hline 108.9 & 20.5 & 38.9 & 25.6 & 27.8 & - & 4.8 & 1.4 & - & 0.6 & 1.0 & $\mathrm{tr}$ \\
\hline 136.1 & 41.9 & 30.9 & 15.2 & 17.1 & 1.4 & 17.4 & 5.2 & 12.6 & - & - & - \\
\hline 136.7 & 45.0 & 30.7 & 13.8 & 20.6 & 0.7 & 23.0 & 5.8 & 5.4 & - & - & $\mathrm{tr}$ \\
\hline 156.1 & 47.3 & 23.4 & 12.9 & 14.6 & 1.7 & 22.4 & 6.2 & 18.1 & - & 0.6 & - \\
\hline 174.4 & 24.8 & 35.4 & 18.6 & 21.2 & 0.8 & 15.2 & 3.8 & 4.0 & - & 0.9 & - \\
\hline \multicolumn{12}{|c|}{$2-20 \mu \mathrm{m}$ Fractions } \\
\hline 12.3 & & 34.6 & 16.2 & 29.5 & - & 12.6 & 4.7 & & 0.5 & 1.9 & $\operatorname{tr}$ \\
\hline 15.4 & & 38.1 & 17.2 & 27.1 & 0.6 & 10.9 & 4.6 & & 0.2 & 1.3 & $\operatorname{tr}$ \\
\hline 49.2 & & 48.4 & 20.3 & 22.9 & - & 5.2 & 2.5 & & - & 0.8 & - \\
\hline 50.2 & & 38.5 & 20.4 & 20.9 & 1.6 & 14.2 & 4.5 & & - & - & - \\
\hline 50.6 & & 38.8 & 17.4 & 26.9 & 0.3 & 11.1 & 3.7 & & 0.6 & 1.2 & tr \\
\hline 79.3 & & 41.0 & 19.6 & 21.7 & 0.5 & 12.3 & 4.3 & & 0.2 & 0.6 & - \\
\hline 108.9 & & 43.0 & 21.9 & 28.1 & - & 3.9 & 1.5 & & 0.2 & 1.3 & $\operatorname{tr}$ \\
\hline 136.1 & & 39.3 & 17.7 & 18.3 & 1.7 & 17.3 & 4.8 & & - & 1.0 & - \\
\hline 136.7 & & 43.1 & 15.5 & 20.5 & - & 15.6 & 4.9 & & 0.3 & - & tr \\
\hline 156.1 & & 40.2 & 16.6 & 21.3 & 1.0 & 15.4 & 5.0 & & - & 0.5 & - \\
\hline 174.4 & & 49.9 & 15.7 & 22.7 & - & 8.0 & 3.2 & & - & 0.6 & - \\
\hline \multicolumn{12}{|c|}{$<2 \mu \mathrm{m}$ Fractions } \\
\hline 12.3 & & 8.4 & 5.5 & 11.5 & - & 14.5 & 6.3 & 52.4 & 0.4 & 1.1 & $\operatorname{tr}$ \\
\hline 15.4 & & 8.9 & 7.3 & 11.4 & 2.5 & 13.5 & 5.3 & 50.5 & 0.2 & 0.4 & tr \\
\hline 49.2 & & 6.7 & 5.4 & 4.6 & 11.1 & 19.6 & 2.2 & 49.4 & 0.4 & 0.6 & $\operatorname{tr}$ \\
\hline 50.2 & & 7.2 & 6.2 & 5.7 & 9.1 & 22.2 & 2.6 & 46.8 & 0.2 & - & - \\
\hline 50.6 & & 7.3 & 4.8 & 7.1 & 1.9 & 15.2 & 3.8 & 59.1 & 0.4 & 0.4 & - \\
\hline 79.3 & & 16.4 & 10.0 & 12.0 & 2.0 & 18.2 & 4.8 & 35.9 & 0.3 & 0.4 & tr \\
\hline 108.9 & & 4.6 & 3.9 & 3.8 & 4.0 & 15.2 & 4.3 & 63.5 & 0.7 & - & - \\
\hline 136.1 & & 6.8 & 7.3 & 5.6 & 11.5 & 22.2 & 2.0 & 44.6 & - & - & - \\
\hline 136.7 & & 12.2 & 9.8 & 10.7 & - & 32.0 & 9.6 & 25.6 & - & - & - \\
\hline 156.1 & & 9.9 & 8.8 & 7.4 & 5.3 & 24.6 & 4.7 & 39.3 & - & - & - \\
\hline 174.4 & & 13.0 & 9.0 & 8.8 & 4.7 & 37.9 & 8.8 & 17.7 & - & - & - \\
\hline
\end{tabular}

${ }^{\mathrm{a}} \mathrm{U}-1$ persistent peak at $9.5 \AA$. Intensity of peak was not observed to be dependent on any other minerals identified. 
TABLE 4

Results of X-Ray Diffraction Analysis From Site 325

\begin{tabular}{|c|c|c|c|c|c|c|c|c|c|c|c|c|c|c|c|c|}
\hline $\begin{array}{c}\text { Sample Depth } \\
\text { Below Sea } \\
\text { Floor }(\mathrm{m})\end{array}$ & Amor. & Calc. & Quar. & Cris. & $\mathrm{K}-\mathrm{Fe}$. & Plag. & Kaol. & Mica & Chlo. & Mont. & Trid. & Clin. & Gibb. & Amph. & $\mathrm{U}-1^{\mathrm{a}}$ & $\mathrm{U}-2^{\mathrm{b}}$ \\
\hline \multicolumn{17}{|l|}{ Bulk Samples } \\
\hline 35.0 & & - & 21.4 & - & 14.1 & 28.9 & & 12.7 & 6.6 & 13.2 & - & 0.7 & & 2.3 & $\mathrm{tr}$ & $\mathrm{tr}$ \\
\hline 35.3 & & - & 20.0 & - & 12.1 & 28.5 & & 16.9 & 6.4 & 12.1 & - & 0.7 & & 2.3 & $\mathrm{tr}$ & $\mathrm{tr}$ \\
\hline 36.8 & & - & 22.0 & - & 10.2 & 30.5 & & 14.2 & 5.8 & 14.6 & - & 0.4 & & 2.4 & $\mathrm{tr}$ & $\mathrm{tr}$ \\
\hline 39.8 & & - & 29.3 & - & 15.7 & 36.7 & & 5.5 & 4.2 & 5.1 & - & 0.5 & & 3.0 & tr & $\mathrm{tr}$ \\
\hline 39.9 & & - & 21.0 & - & 6.0 & 37.9 & & 7.0 & 6.0 & 17.0 & - & 0.4 & & 4.7 & $\mathrm{tr}$ & $\mathrm{tr}$ \\
\hline 169.4 & & - & 24.5 & - & 10.4 & 36.9 & & 6.5 & 7.0 & 10.5 & - & 0.8 & & 3.4 & $\mathrm{tr}$ & $\mathrm{tr}$ \\
\hline 178.9 & & - & 22.5 & - & 10.5 & 37.1 & & 7.4 & 6.0 & 12.4 & - & 1.1 & & 3.1 & $\mathrm{tr}$ & $\mathrm{tr}$ \\
\hline 180.0 & & - & 27.1 & - & 16.5 & 38.8 & & 3.5 & 5.0 & 4.9 & - & 0.5 & & 3.7 & $\mathrm{tr}$ & $\operatorname{tr}$ \\
\hline 182.2 & & - & 25.2 & - & 14.0 & 39.4 & & 4.9 & 6.0 & 6.1 & - & 0.4 & & 4.1 & $\mathrm{tr}$ & $\mathrm{tr}$ \\
\hline 520.0 & & - & 13.9 & 28.2 & 15.3 & 26.0 & & 1.9 & 3.0 & 9.5 & - & 1.4 & & 0.8 & $\mathrm{tr}$ & - \\
\hline 614.0 & & 4.4 & 14.6 & 21.6 & 7.2 & 22.8 & & 4.0 & 4.1 & 18.6 & - & 1.8 & & 0.8 & - & $\mathrm{tr}$ \\
\hline 615.1 & & 13.7 & 12.8 & 18.3 & 16.9 & 18.9 & & 5.1 & 2.0 & 10.2 & - & 1.5 & & 0.7 & - & $\mathrm{tr}$ \\
\hline 616.8 & & - & 9.5 & 25.3 & 6.3 & 12.0 & & 35.0 & 1.4 & 6.0 & 2.0 & 2.3 & & 0.2 & - & tr \\
\hline 643.6 & & - & 23.8 & - & 17.6 & 34.4 & & 1.5 & 1.3 & 7.2 & - & 13.6 & & 0.6 & - & $\mathrm{tr}$ \\
\hline 644.9 & & - & 15.2 & - & 15.8 & 28.1 & & 8.0 & 4.7 & 22.5 & - & 4.4 & & 1.3 & - & $\mathrm{tr}$ \\
\hline 710.5 & & - & 6.6 & - & 14.2 & 22.4 & & - & 0.7 & 51.9 & - & 3.7 & & 0.5 & - & $\mathrm{tr}$ \\
\hline 712.2 & & - & 22.7 & - & 19.7 & 32.4 & & 2.9 & 1.7 & 5.4 & - & 14.7 & & 0.6 & - & tr \\
\hline \multicolumn{17}{|c|}{$2-20 \mu \mathrm{m}$ Fractions } \\
\hline 35.0 & & & 32.7 & - & 17.2 & 32.5 & - & 7.2 & 6.2 & - & & 0.8 & & 3.3 & $\mathrm{tr}$ & $\operatorname{tr}$ \\
\hline 35.3 & & & 25.3 & - & 17.9 & 35.4 & - & 9.8 & 6.9 & - & & 0.7 & & 4.1 & tr & $\mathrm{tr}$ \\
\hline 36.8 & & & 28.4 & - & 15.5 & 32.2 & - & 12.5 & 7.2 & - & & 0.9 & & 3.5 & $\mathrm{tr}$ & $\mathrm{tr}$ \\
\hline 39.8 & & & 29.5 & - & 17.1 & 35.1 & - & 7.1 & 5.8 & - & & 0.9 & & 4.4 & $\mathrm{tr}$ & $\mathrm{tr}$ \\
\hline 39.9 & & & 23.9 & - & 20.6 & 35.5 & - & 3.7 & 3.7 & 7.0 & & 0.3 & & 5.2 & $\mathrm{tr}$ & - \\
\hline 169.4 & & & 32.0 & - & 16.2 & 39.9 & - & 2.0 & 5.0 & - & & 0.8 & & 4.1 & $\mathrm{tr}$ & $\mathrm{tr}$ \\
\hline 178.9 & & & 26.0 & - & 24.2 & 33.6 & - & 5.2 & 6.0 & - & & 1.1 & & 4.0 & $\mathrm{tr}$ & tr \\
\hline 180.0 & & & 29.8 & - & 16.6 & 40.6 & - & 3.9 & 4.0 & - & & 0.8 & & 4.4 & tr & $\operatorname{tr}$ \\
\hline 182.2 & & & 26.0 & - & 17.9 & 42.0 & - & 4.3 & 4.2 & - & & 0.6 & & 5.0 & tr & tr \\
\hline 520.0 & & & 17.4 & 34.4 & 11.4 & 26.9 & 0.3 & 2.2 & 2.3 & 3.4 & & 0.9 & & 0.9 & $\mathrm{tr}$ & tr \\
\hline 614.0 & & & 20.3 & 19.1 & 20.4 & 25.3 & - & 5.4 & 4.0 & 2.5 & & 1.9 & & 1.1 & - & tr \\
\hline 615.1 & & & 22.5 & 14.2 & 19.9 & 30.0 & - & 5.1 & 2.6 & 2.7 & & 2.1 & & 0.9 & - & $\mathrm{tr}$ \\
\hline 616.8 & & & 21.7 & 30.4 & 14.4 & 18.4 & - & 6.7 & 3.3 & 3.1 & & 1.2 & & 0.8 & - & $\mathrm{tr}$ \\
\hline 643.6 & & & 11.1 & - & 27.2 & 39.0 & - & 3.0 & 1.1 & - & & 18.2 & & 0.3 & - & tr \\
\hline 644.9 & & & 25.3 & - & 35.0 & 15.7 & - & 6.7 & 5.2 & 7.1 & & 3.3 & & 1.8 & - & $\mathrm{tr}$ \\
\hline 710.5 & & & 8.2 & - & 48.7 & 30.7 & - & 2.2 & 1.3 & - & & 8.5 & & 0.5 & - & $\mathrm{tr}$ \\
\hline 712.2 & & & 21.9 & - & 26.9 & 33.2 & - & 4.7 & 2.2 & - & & 10.0 & & 1.2 & - & $\mathrm{tr}$ \\
\hline \multicolumn{17}{|c|}{$<2 \mu \mathrm{m}$ Fractions } \\
\hline 35.0 & 49.3 & & 8.5 & - & 10.2 & 11.9 & - & 14.2 & 12.7 & 39.8 & - & 1.5 & - & 1.2 & - & - \\
\hline 35.3 & 47.1 & & 8.8 & - & 8.9 & 16.0 & 2.3 & 15.6 & 9.3 & 37.8 & - & - & - & 1.3 & - & - \\
\hline 36.8 & 39.7 & & 9.4 & - & 7.1 & 9.2 & 3.7 & 13.2 & 5.8 & 49.3 & - & 0.9 & - & 1.4 & - & - \\
\hline 39.8 & 31.3 & & 10.1 & - & 12.1 & 12.6 & - & 13.5 & 15.0 & 34.5 & - & 0.9 & - & 1.4 & $\mathrm{tr}$ & - \\
\hline 39.9 & 49.1 & & 8.0 & - & 9.3 & 14.2 & 1.6 & 8.7 & 7.7 & 48.7 & - & - & - & 1.8 & $\mathrm{tr}$ & - \\
\hline 169.4 & 49.3 & & 11.2 & - & 7.2 & 14.8 & 3.1 & 6.7 & 10.9 & 43.7 & - & 0.9 & - & 1.4 & - & - \\
\hline 178.9 & 44.1 & & 10.3 & - & 6.7 & 12.5 & - & 8.1 & 12.0 & 47.7 & - & 1.0 & - & 1.7 & tr & - \\
\hline 180.0 & 42.7 & & 10.6 & - & 13.5 & 13.6 & - & 12.0 & 14.2 & 33.6 & - & 0.4 & - & 2.1 & $\mathrm{tr}$ & - \\
\hline 182.2 & 45.3 & & 12.6 & - & 7.3 & 20.0 & - & 8.2 & 15.8 & 32.4 & - & 1.1 & - & 2.6 & tr & - \\
\hline 520.0 & 18.4 & & 4.3 & 48.7 & 4.4 & 10.5 & - & 3.1 & 3.1 & 22.9 & 2.4 & 0.8 & - & - & - & - \\
\hline 614.0 & 50.9 & & 9.8 & - & 11.8 & 15.4 & - & 9.0 & 6.7 & 44.9 & - & 1.2 & - & 1.3 & - & - \\
\hline 615.1 & 33.9 & & 7.8 & 13.8 & 7.3 & 20.2 & 1.0 & 7.6 & 2.3 & 39.1 & - & 1.0 & - & - & - & $\mathrm{tr}$ \\
\hline 616.8 & 38.7 & & 8.9 & 29.5 & 8.1 & 9.5 & - & 10.2 & 4.4 & 28.5 & - & 0.9 & - & - & - & - \\
\hline 643.6 & 32.5 & & 35.9 & - & 10.6 & 14.6 & 0.6 & 7.4 & 4.4 & 13.9 & - & 11.6 & 0.5 & 0.6 & - & $\mathrm{tr}$ \\
\hline 644.9 & 39.3 & & 17.6 & - & 6.4 & 15.3 & - & 7.5 & 6.7 & 44.5 & - & 1.9 & - & - & - & - \\
\hline 710.5 & 1.2 & & 3.4 & - & 4.5 & 7.3 & - & 1.6 & 2.3 & 79.8 & - & 1.2 & - & - & - & - \\
\hline 712.2 & 28.8 & & 34.8 & - & 9.7 & 14.5 & 0.5 & 10.8 & 5.2 & 20.1 & - & 3.9 & - & 0.6 & - & - \\
\hline
\end{tabular}

${ }^{a} \mathrm{U}-1$ persistent peak at $9.5 \AA$. Intensity of peak was not observed to be dependent on any other minerals identified.

${ }^{b} \mathrm{U}-2$ peak at $2.53 \AA$, possibly magnetite. Optical examination indicates its presence, but secondary peaks were not detected. 
TABLE 5

Samples Submitted for X-Ray Diffraction Analysis, Leg 35

\begin{tabular}{|c|c|c|c|c|}
\hline $\begin{array}{c}\text { Sample } \\
\text { (Interval in } \mathrm{cm} \text { ) }\end{array}$ & $\begin{array}{c}\text { Depth Below } \\
\text { Sea Floor } \\
(\mathrm{m})\end{array}$ & GSA Color & $\begin{array}{c}\text { GSA Color } \\
\text { Code } \\
\text { Number }\end{array}$ & Sediment Description \\
\hline \multicolumn{5}{|l|}{ Site 322} \\
\hline $1-4,82-84$ & 81.8 & Grayish-green & $10 \mathrm{GY} 5 / 2$ & $\begin{array}{l}\text { Mica bearing quartz rich muddy } \\
\text { sandstone }\end{array}$ \\
\hline $3-1,20-22$ & 295.2 & Dusky yellow-green & 5 GY $5 / 2$ & Bioturbated sandy claystone \\
\hline $4-1,65-69$ & 352.7 & Dark greenish-gray & 5 GY $4 / 1$ & $\begin{array}{l}\text { Muddy sand interlaminated with } \\
\text { sandy clay }\end{array}$ \\
\hline $4-2,110-112$ & 354.6 & Grayish-green & $10 \mathrm{GY} 5 / 2$ & Mudstone \\
\hline $5-1,76-77$ & 391.8 & Dark greenish-gray & $5 \mathrm{G} 4 / 1$ & Mudstone \\
\hline $9-2,140-142$ & 469.9 & Dusky brown & $5 \mathrm{YR} 2 / 2$ & Sandstone \\
\hline $10-1,49-50$ & 487.0 & Olive-gray & $5 \times 3 / 2$ & Clay \\
\hline $10-1,106-107$ & 487.6 & Olive-gray & $5 \mathrm{Y} 3 / 2$ & Sandstone \\
\hline $11-4,98-100$ & 510.5 & Dusky yellowish-brown & $10 \mathrm{YR} 2 / 2$ & Clay \\
\hline $11-5,88-90$ & 511.9 & Dark yellowish-brown & $10 \mathrm{YR} 4 / 2$ & Clay \\
\hline $11-6,46-48$ & 513.0 & Grayish-brown & $5 \mathrm{YR} 3 / 2$ & Fe-Mn colloid bearing clay \\
\hline $11-6,129-130$ & 513.8 & Moderate brown & $5 \mathrm{YR} 3 / 4$ & Fe-Mn colloid bearing clay \\
\hline \multicolumn{5}{|l|}{ Site 323} \\
\hline $1-1,107-108$ & 76.6 & Medium light gray & N6 & Clayey sandstone \\
\hline $1-3,75-76$ & 79.3 & Light olive-gray & $5 \mathrm{Y} 6 / 1$ & Clay \\
\hline $2-1,83-84$ & 161.8 & Yellowish-gray & $5 Y 7 / 2$ & Clay \\
\hline $2-1,119-120$ & 162.2 & Pale olive & $10 \mathrm{Y} 6 / 2$ & Clay \\
\hline $3-2,119-120$ & 258.7 & Greenish-gray & $5 \mathrm{G} 6 / 1$ & Clayey sandstone \\
\hline $3-2,136-137$ & 258.9 & Pale olive & $10 \mathrm{Y} 6 / 2$ & Clay \\
\hline $5-1,140-141$ & 333.4 & Grayish-orange & $10 \mathrm{YR} 7 / 4$ & $\begin{array}{l}\text { Manganese micronodule bearing } \\
\text { clay }\end{array}$ \\
\hline \multirow[t]{2}{*}{$6-1,140-141$} & 341.5 & Pale yellowish-brown & $10 \mathrm{YR} 6 / 2$ & Mud \\
\hline & & Pale olive & 10 Y $6 / 2$ & Sandy mud \\
\hline $6-1,140-141$ & 341.5 & Grayish-orange & 10 YR $7 / 4$ & Fe-Mn colloid bearing clay \\
\hline $6-1,140-141$ & 341.5 & Olive-gray & $5 \mathrm{Y} 4 / 1$ & Fe-Mn colloid bearing clay \\
\hline $6-1,135-137$ & 342.9 & Yellowish-gray & $5 \times 7 / 2$ & Biosiliceous clay \\
\hline $6-1,147-148$ & 343.0 & Pale yellowish-brown & $10 \mathrm{YR} 6 / 2$ & Clay \\
\hline $7-3,5-6$ & 363.6 & Pale grayish-red & $5 \mathrm{R} 5 / 2$ & Mud \\
\hline $8-1,106-107$ & 409.1 & Pinkish-gray & 5 YR $8 / 1$ & Sandy mud \\
\hline $8-1,117-118$ & 409.2 & Dusky yellowish-brown & $10 \mathrm{YR} 2 / 2$ & Siliceous argilite \\
\hline $8-1,143-148$ & 409.4 & Grayish-olive green & $5 \mathrm{GY} 4 / 2$ & Biosiliceous mud \\
\hline $9-2,99-100$ & 458.0 & Grayish-green & $10 \mathrm{GY} 5 / 2$ & Biosiliceous mud \\
\hline $9-2,131-132$ & 458.3 & Dusky green & $5 \mathrm{G} 3 / 2$ & Siliceous argillite \\
\hline $10-1,122-126$ & 504.2 & Grayish-green & $10 \mathrm{GY} 5 / 2$ & Mud \\
\hline $10-2,117-120$ & 505.7 & Grayish-green & $10 \mathrm{GY} 5 / 2$ & Mud \\
\hline $10-3,125-127$ & 507.3 & Grayish-green & $10 \mathrm{GY} 5 / 2$ & Mud \\
\hline $11-1,70-72$ & 551.2 & Grayish-green & $5 \mathrm{G} 5 / 2$ & Mud \\
\hline $12-2,71-72$ & 600.2 & Dusky yellow-green & $5 \mathrm{GY} 5 / 2$ & Sandy mud \\
\hline $12-2,78-80$ & 600.3 & Grayish-green & $10 \mathrm{GY} 5 / 2$ & Sandy mud \\
\hline $12-2,106-108$ & 600.6 & Greenish-gray & $5 \mathrm{G} 6 / 1$ & Sandy mud \\
\hline $13-5,123-125$ & 624.2 & Dark yellowish-brown & $10 \mathrm{YR} 4 / 2$ & Sandy mud \\
\hline $13-6,92-94$ & 625.4 & Dark greenish-gray & $5 \mathrm{G} 5 / 1$ & Sandy mud \\
\hline $14-2,93-97$ & 638.4 & Dusky yellowish-brown & $10 \mathrm{YR} 2 / 2$ & Fe-Mn colloid bearing mud \\
\hline $15-1,132-134$ & 656.6 & Dusky yellowish-brown & $10 \mathrm{YR} 2 / 2$ & Fe-Mn colloid bearing mud \\
\hline $15-2,114-117$ & 657.9 & Dusky yellowish-brown & $10 \mathrm{YR} 2 / 2$ & Fe-Mn colloid bearing mud \\
\hline $15-3,121-124$ & 659.5 & Grayish-brown & $5 \mathrm{YR} 3 / 2$ & $\begin{array}{l}\text { Fe-Mn colloid and volcanic } \\
\text { glass bearing mud }\end{array}$ \\
\hline
\end{tabular}


TABLE 5 - Continued

\begin{tabular}{|c|c|c|c|c|}
\hline $\begin{array}{c}\text { Sample } \\
\text { (Interval in cm) }\end{array}$ & $\begin{array}{l}\text { Depth Below } \\
\text { Sea Floor } \\
(\mathrm{m})\end{array}$ & GSA Color & $\begin{array}{l}\text { GSA Color } \\
\text { Code } \\
\text { Number }\end{array}$ & Sediment Description \\
\hline $15-4,95-98$ & 660.7 & Dusky yellowish-brown & $10 \mathrm{YR} 2 / 2$ & Fe-Mn colloid bearing clay \\
\hline $15-5,111-115$ & 662.4 & Dusky yellowish-brown & $10 \mathrm{YR} 2 / 2$ & Fe-Mn colloid bearing clay \\
\hline $15-6,69-72$ & 663.5 & Grayish-brown & $5 \mathrm{YR} 3 / 2$ & $\begin{array}{l}\text { Fe-Mn colloid bearing calcareous } \\
\text { rich clay }\end{array}$ \\
\hline $16-1,90-94$ & 665.4 & Pale brown & 5 YR $5 / 2$ & $\begin{array}{l}\text { Fe-Mn colloid bearing calcareous } \\
\text { ooze }\end{array}$ \\
\hline $16-3,31-33$ & 667.8 & Moderate brown & 5 YR $4 / 4$ & Fe-Mn colioid bearing clay \\
\hline $16-4,73-74$ & 669.7 & Dark yellowish-brown & $10 \mathrm{YR} 4 / 2$ & Fe-Mn colloid bearing clay \\
\hline $18-2,116-118$ & 695.7 & Dark yellowish-brown & 10 YR $4 / 2$ & Fe-Mn colloid bearing mud \\
\hline $18-3,99-101$ & 697.0 & Moderate yellowish-brown & 10 YR $5 / 4$ & Fe-Mn colloid bearing clay \\
\hline $18-4,52-54$ & 698.0 & $\begin{array}{l}\text { Dusky yellowish-brown } \\
\text { Dark yellowish-brown }\end{array}$ & $\begin{array}{l}10 \text { YR } 2 / 2 \\
10 \text { YR } 4 / 2\end{array}$ & Fe-Mn colloid bearing mud \\
\hline $18-5,0-2$ & 699.0 & Moderate brown & 5 YR $3 / 4$ & Fe-Mn colloid bearing clay \\
\hline Site 324 & & & & \\
\hline $1-3,27-29$ & 12.3 & Pale yellowish-brown & $10 \mathrm{YR} 6 / 2$ & Clay \\
\hline $1-5,40-46$ & 15.4 & Pale yellowish-brown & $10 \mathrm{YR} 6 / 2$ & Clay \\
\hline $2-2,18-21$ & 49.2 & Light olive-gray & 5 Y $6 / 1$ & Mud \\
\hline $2-2,120-125$ & 50.2 & Grayish-olive & $10 \mathrm{Y} 4 / 2$ & Mud \\
\hline $2-3,6-11$ & 50.6 & Light olive-gray & $5 \times 5 / 2$ & Rock fragment bearing sandy mud \\
\hline $3-3,81-86$ & 79.3 & Olive-gray & 5 Y $4 / 1$ & Quartz and feldspar rich mud \\
\hline $4-4,40-41$ & 108.9 & Dusky yellowish-green & 5 GY $5 / 2$ & Quartz rich silty sand \\
\hline & 136.1 & Dark yellowish-brown & $10 \mathrm{YR} 4 / 2$ & Mud \\
\hline & 136.7 & Grayish-olive-green & 5 GY $3 / 2$ & Quartz and feldspar bearing mud \\
\hline $7-3,137-141$ & 156.1 & Dark greenish-gray & 5 GY $4 / 1$ & $\begin{array}{l}\text { Calcareous bearing quartz and } \\
\text { feldspar rich mud }\end{array}$ \\
\hline $8-3,91-92$ & 174.4 & Dark greenish-gray & $5 \mathrm{G} 4 / 1$ & $\begin{array}{l}\text { Quartz rich feldspar and mafic } \\
\text { mineral bearing silt }\end{array}$ \\
\hline Site 325 & & & & \\
\hline $1-1,103-106$ & 35.0 & Dark greenish-gray & $5 \mathrm{G} 4 / 1$ & Calcareous bearing mud \\
\hline $1-1,130-132$ & 35.3 & Dark greenish-gray & $5 \mathrm{G} 4 / 1$ & Calcareous bearing mud \\
\hline $1-2,131-134$ & 36.8 & Olive-gray & $5 \times 4 / 1$ & Quartz rich mud \\
\hline $1-4,131-133$ & 39.8 & Dark greenish-gray & $5 \mathrm{G} 4 / 1$ & $\begin{array}{l}\text { Calcareous bearing metamorphic } \\
\text { rock fragment bearing sandy mud }\end{array}$ \\
\hline $1-4,143-144$ & 39.9 & Light olive-gray & $5 \times 5 / 2$ & Quartz and feldspar bearing mud \\
\hline $2-2,93-95$ & 169.4 & Olive-gray & $5 \times 4 / 1$ & Calcite bearing mud \\
\hline $3-2,90-92$ & 178.9 & Dark greenish-gray & 5 GY $4 / 1$ & Calcareous bearing mud \\
\hline $3-3,51-54$ & 180.0 & Dark greenish-gray & $5 \mathrm{GY} 4 / 1$ & $\begin{array}{l}\text { Calcareous metamorphic rock } \\
\text { fragment bearing sandy mud }\end{array}$ \\
\hline $3-4,121-123$ & 182.2 & Dark greenish-gray & $5 \mathrm{GY} 4 / 1$ & $\begin{array}{l}\text { Calcareous metamorphic rock } \\
\text { fragment bearing heavy mineral } \\
\text { bearing sandy mud }\end{array}$ \\
\hline $7-2,0-2$ & 520.0 & Dark greenish-gray & $5 \mathrm{GY} 4 / 1$ & Mudstone \\
\hline $8-1,147-149$ & 614.0 & Olive-gray & $5 \mathrm{Y} 4 / 1$ & Calcareous claystone \\
\hline $8-2,112-113$ & 615.1 & Olive-gray & $5 Y 4 / 1$ & Calcareous mudstone \\
\hline $8-3,130-133$ & 616.8 & Dark greenish-gray & 5 GY $4 / 1$ & Calcareous bearing claystone \\
\hline $9-2,106-110$ & 643.6 & $\begin{array}{l}\text { Dark greenish-gray } \\
\text { Medium gray }\end{array}$ & $\begin{array}{l}5 \text { GY } 4 / 1 \\
\text { N } 5\end{array}$ & Silty sand \\
\hline $9-3,92-95$ & 644.9 & Dark greenish-gray & $5 \mathrm{G} 4 / 1$ & Mudstone \\
\hline $10-2,46-48$ & 710.5 & Light olive-gray & $5 \times 5 / 2$ & $\begin{array}{l}\text { Metamorphic rock fragment } \\
\text { feldspar and quartz rich silty } \\
\text { sandstone }\end{array}$ \\
\hline $10-3,71-73$ & 712.2 & \multicolumn{2}{|c|}{$\begin{array}{l}\text { Well laminated sample ranging in color } \\
\text { from very light gray } \mathrm{N} 8 \text {, to dark } \\
\text { greenish gray } 5 \text { GY } 4 / 1\end{array}$} & Silty sand and muddy sand \\
\hline
\end{tabular}

